\title{
Automatic data processing based on the skewness statistic parameter for subsurface defect detection by active infrared thermography
}

\author{
by F. J. Madruga*, C. Ibarra-Castanedo**, O.M. Conde*, J.M. López-Higuera* and X. Maldague** \\ * Photonic Engineering Group, University of Cantabria, E.T.S.I.I.T. Av. De Los Castros s/n 39005 Santander \\ Cantabria, Spain; (francisco.madruga,olga.conde, miguel.lopezhiguera)@unican.es \\ ${ }^{* *}$ Computer Vision and Systems Laboratory, Department of Electrical and Computer Engineering, 1065, Av. de la \\ Médecine, Laval University, Quebec City, Canada, G1V 0A6; (IbarraC, MaldagX)@gel.ulaval.ca
}

\section{Abstract}

Non-destructive testing (NDT) by active infrared thermography requires of data processing techniques in order to: $i$. improve the thermal contrast between defective and non-defective areas, and ii. reduce the large quantity of images recorded during the inspection without losing relevant information. In this paper, the use of the skewness statistic parameter is proposed for the automatic processing of thermographic sequences obtained by active thermography. The main interest is to compress data from a 3D thermogram sequence to a unique skewness parameter image containing all the relevant information about the subsurface defects. Experimental thermographic data from three CFRP specimens with different surface shape (planar, curved and trapezoidal), containing 25 artificial defects (Teflon ${ }^{\circledR}$ inserts) at different depths and having several sizes, have been processed using the skewness parameter. The results presented herein demonstrate the potential of this technique for automatic defect detection by active thermography.

\section{Introduction}

Active infrared thermography applied to the non-destructive testing (NDT) of materials offers a reliable, straightforward and fast means for retrieving structural information from a specimen. The technique is based on the detection of surface temperature anomalies that appear in response to the application of a thermal pulse to the specimen surface. However, active thermography presents some limitations fundamentally due to an exponential rate of attenuation of the defect signature with its depth - a consequence of the dependence of thermography on the heat conduction processes to convey information about internal structural anomalies. Defect enhancement techniques must be applied in order to produce a successful thermal inspection [1]. Conventional image processing techniques such as: the time-derivative approach by thermographic signal reconstruction (TSR) [2], the automatic algorithm based on differential absolute contrast (DAC) [3], and the application of the Fourier transform, known as pulsed phase thermography (PPT) [4] , have been applied to pulsed thermographic inspection showing both advantages and disadvantages. Recently, principal component analysis (PCA), based on the second order statistic of data, was introduced in thermal NDT. This technique called principal component thermography (PCT) [5], besides of showing some potentialities for defect detection, it compresses the information contained in the sequence to a fewer number of PCs, the number of components being dependent on the amount and type of defects and the experimental conditions and requiring an operator's decision. The present work describes an alternative method based on the third order statistic of data called skewness, which can be used to compress the information contained in the $3 \mathrm{D}$ sequence into a single image. The performance of the proposed method has been evaluated for depth characterization on three carbon fibre reinforced plastic (CFRP) specimens containing several defects, having different surface geometries, and being subjected to uniform and non-uniform excitation.

\section{Basic principles of skewness.}

Skewness is the third standardized moment of a distribution. The term moment is employed to represent the expected valued of different powers of a random variable. Skewness is not employed for descriptive purposes as frequently as the mean and the variance. It is employed within the context of determining the goodness of fit of data in reference to a specific type of distribution. Mathematically, skewness is calculated from [6]:

$$
k_{3}(x)=\frac{E\left[[x-\mu]^{3}\right]}{\sigma^{3}}
$$

where $\mu$ and $\sigma$ are the mean and standard deviation of random variable $x$, respectively and $E[]$ is the mathematic expectancy defined as

$$
E[X]=\frac{1}{P} \sum_{n=1}^{P} x_{n}
$$

where $X=\left[\begin{array}{lllll}x_{1} & x_{2} & x_{3} & \ldots & x_{P}\end{array}\right]$ is a $T X P$ dimensional matrix and $P$ point $x_{\mathrm{n}}$ are the $T$-dimension. 
Table 1 Example of symmetrical (Distribution A) and asymmetrical (distributions $B$ and C) distributions.

\begin{tabular}{|l|l|l|l|l|}
\hline Distribution & Distribution Value & Mean & Variance & Skewness \\
\hline A & $-4,-2,-2,0,0,0,0,2,2,4$ & 0 & 5.3333 & 0 \\
\hline B & $-4,-4,-4,-2,-2,0,0,0,2,4$ & -1 & 7.3333 & 0.4246 \\
\hline C & $-4,-2,0,0,0,2,2,4,4,4$ & 1 & 7.3333 & -0.4246 \\
\hline
\end{tabular}
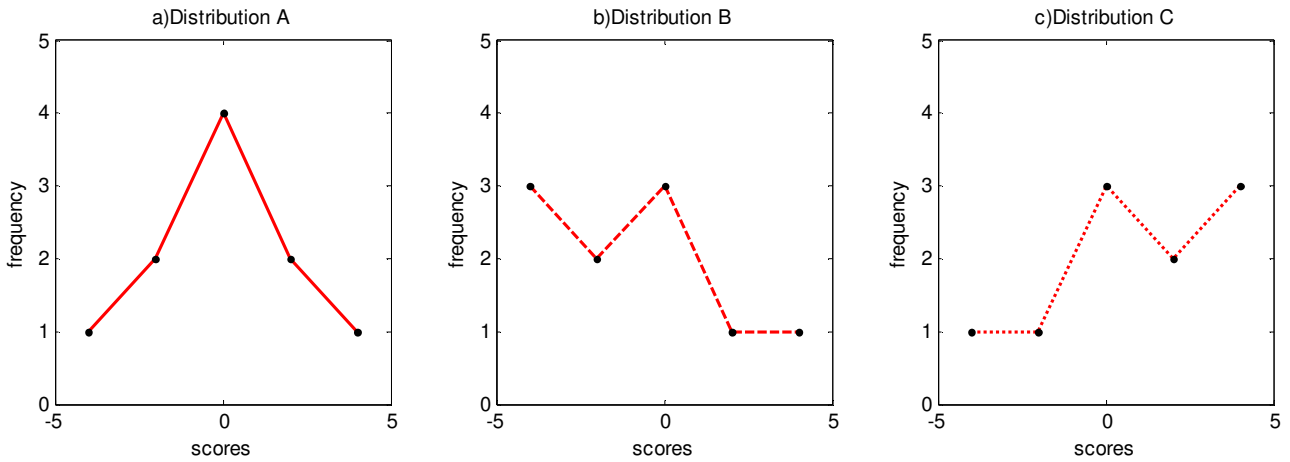

Fig 1. Example of a) symmetrical, b) positively skewed, c) negatively skewed distributions

Skewness is a measure of the asymmetry of the probability distribution of a real-valued random variable [7]. A distribution is symmetrical if it shows two identical mirror images when it is split down the middle as the normal distribution. When a distribution is not symmetrical, a scores fall disproportionally either to the left or right of the middle of distribution. To illustrate the above, three distributions $\mathrm{A}, \mathrm{B}$ y $\mathrm{C}$ have been considered in figure 1. Their mean, variance and skewness values have been calculated and shown in Table 1. The distribution A represented in figure 1 a) shows a symmetrical distribution and its skewness value is zero. Two asymmetrical distributions, $B$ and $C$, are shown in the figure $1 \mathrm{~b}$ ) and $1 \mathrm{c}$ ), being positively and negatively skewed, respectively.

The distribution $A$ is a unimodal symmetrical distribution. The number of scores in its left and right tail is identical. The distribution B is positively skewed. As the tail of the distribution is on the right side it is sometimes called skewed to the right. The distribution $\mathrm{C}$ is negatively skewness or skewed to left.

\section{Skewness measure in pulsed thermography}

In order to apply the skewness measure for subsurface defect detection by pulsed thermography, the setup depicted in figure 2 was used. The surface temperature temporal evolution after a heat excitation was recorded into I images ( $n_{x} \times n_{y}$ pixels), resulting on a 3D matrix. In order to apply Eq. (1), a pre-processing step is needed.

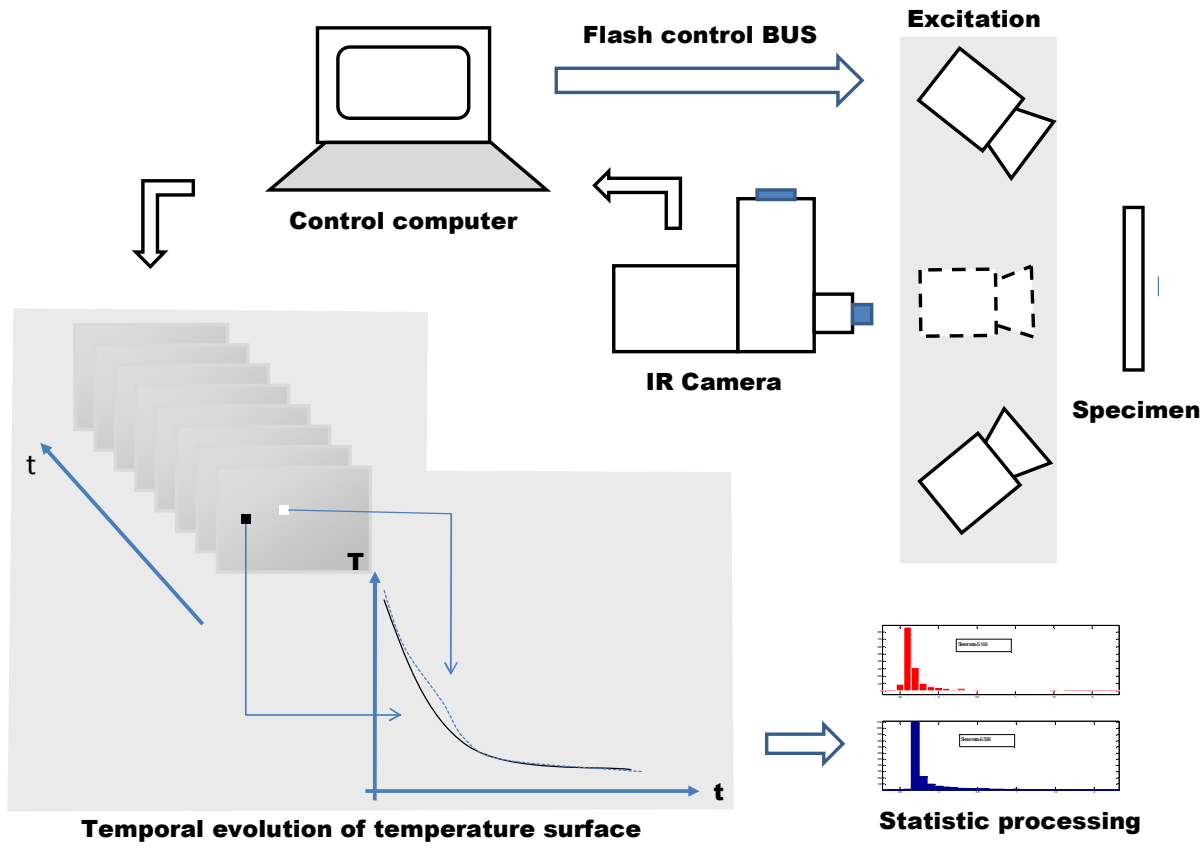

Fig 2. Data acquisition and processing system implemented. 

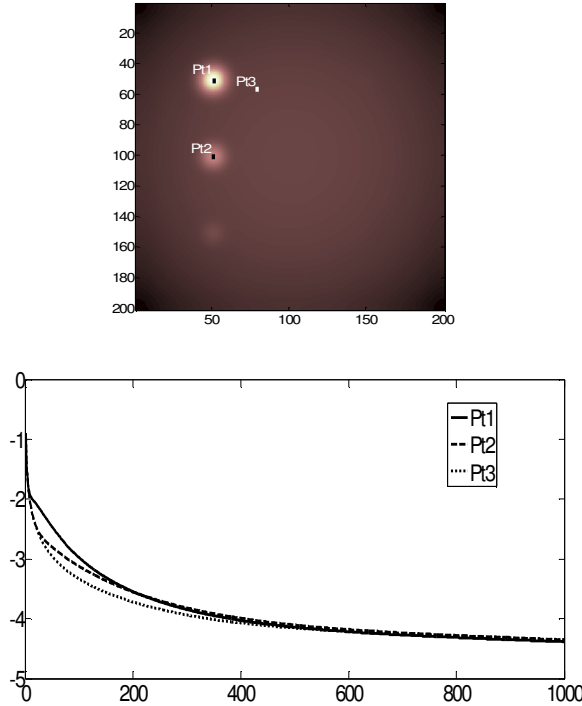

a)

Fig 3. a) Surface temperature evolution of three pixels (Pt1, Pt2 \& Pt3) and b) their histograms
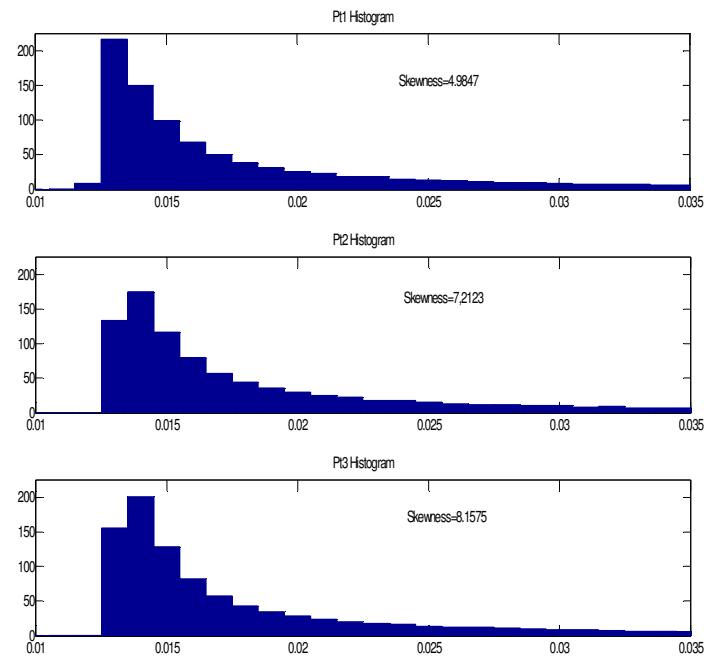

b) contains an image ( $P=n_{x} \times n_{y}$ dimension vector) and each row contains the temporal evolution of each surface pixel (I dimension vector).

To calculate the skewness each row is considered as the scores of the distribution. Figure 3a) shows the temporal evolution of three pixels obtained from 1000 images generated by Thermocal ${ }^{\circledR}$ [8]and a themogram. Two pixels (Pt1 and Pt2) correspond to defects at different depths and Pt3 corresponds to a sound area. Their histogram distributions are shown en figure 3b). All distributions are asymmetrical and skewed to right, that is they are positively skewed and their skewness parameter has a positive value. The skewness value is different for each point. Sound area pixels (Pt3) present the highest skewness values. For defected pixels, the skewness value increases with the defect depth.

In summary, the skewness value of a data distribution obtained from the surface temperature evolution depends on both the subsurface defect presence and its depth.

\section{Experimental setup and data acquisition}

In order to demonstrate the above, three CFRP specimens $(300 \times 300 \times 2 \mathrm{~mm})$ were tested in reflection mode using two high-power photographic flashes $(6.4 \mathrm{~kJ}$ for $5 \mathrm{~ms})$. Each specimen was excited using either 1 flash or 2 flashes for estimating the performance of the method in non-uniform heating conditions. In figure 2 , the two configurations of flashes are illustrated. The flash position used for single excitation is drawn with a dotted line. The surface cooling down process was recorded with a medium wave IR camera ( 3 to $5 \mu \mathrm{m})$ at a frequency of $157 \mathrm{~Hz}$.

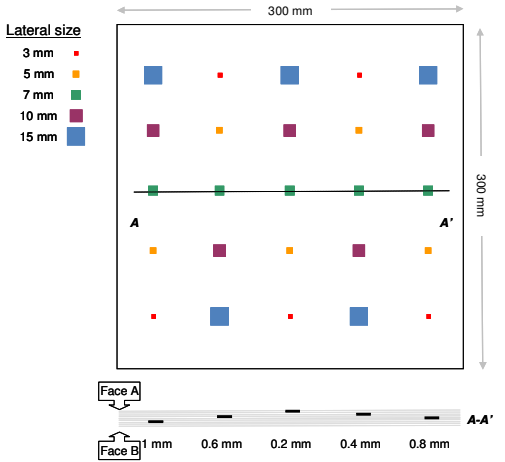

(a)

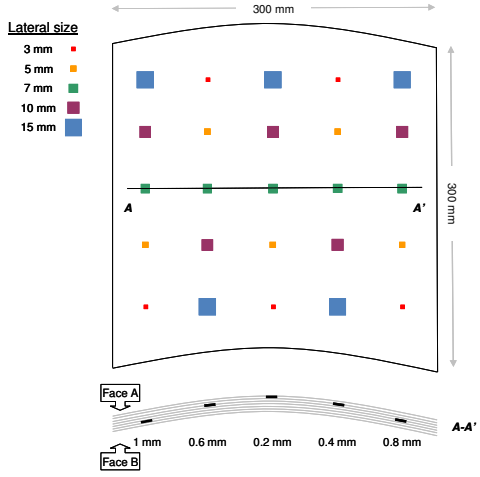

(b)

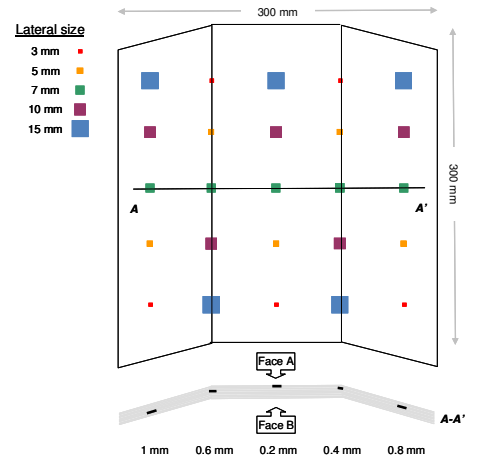

(c)

Fig 4. Schematic representation and defect location for specimen a) CFRP006, b) CFRP007, and c) CFRP008. 
The thermogram matrices contained 1896 thermograms including cold and saturated thermograms. Each specimen contained a total of 25 Teflon $^{\circledR}$ square inclusions having 5 lateral sizes $(3,5,7,10$ and $15 \mathrm{~mm})$ and 5 depths $(0.2,0.4,0.6,0.8$ and $1 \mathrm{~mm}$ when inspected from side A. Figure 4 presents the geometry and defect distribution on the (a) planar, (b) curved and (c) trapezoidal plates.

\section{Results}

The figure 3 shows the skewness parameter images obtained from the three measured specimens (planar, curved and trapezoidal) using a two-flashes excitation in reflection mode on face A. Figure 3a) for planar specimen shows that 23 of 25 defects have been detected on a unique image. For the other case, 22 of 25 defects have been detected. As can be seen, the specimen's shape has little impact on the results. Only the deepest and smallest defects were not detected. The net structure of carbon fibres is highlighted, displaying the anisotropic nature of CFRP, presenting the highest thermal conductivity in the longitudinal direction of fibre.

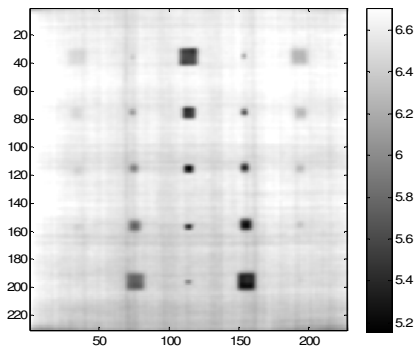

(a)

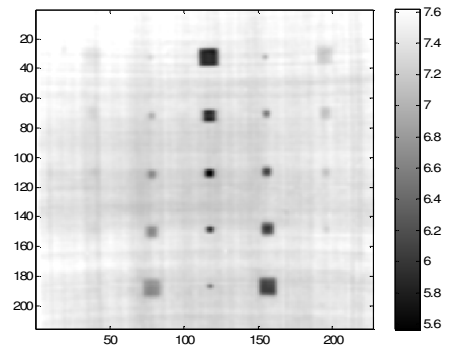

(b)

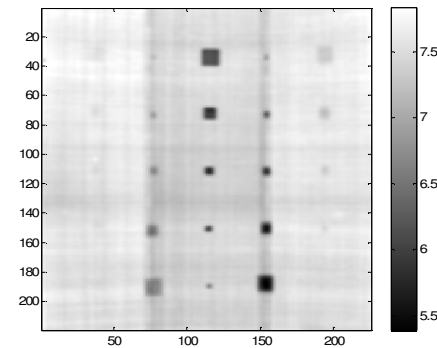

(c)

Fig 5. Unique skewness parameter image (a) CFRP006, (b) CFRP007, and (c) CFRP008

The influence of non-uniform excitation in the processing method can be evaluated in figure 6 for specimens CFRP006 and CFRP008. This results show that, even though defects are detected with practically no difference in contrast compared to figure $5 \mathrm{a}$ ) and c), the impact of non-uniform heating is obvious in the lower part of the image center in figure $6 \mathrm{a}$ ) however it is inappreciable in figure $6 \mathrm{~b}$ ).

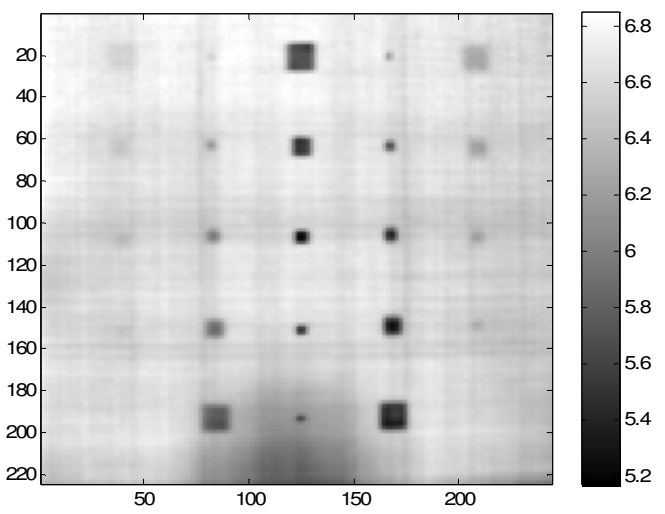

(a)

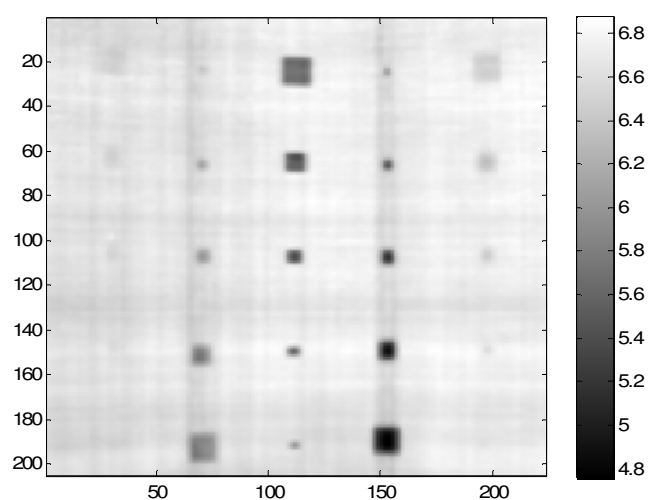

(b)

Fig 6. Unique skewness parameter image (a) CFRP006, b) CFRP008 using one flash excitation focused at a point (bottom-center)

The skewness parameter image presents a good defect-sound area contrast but the minimum and maximum skewness values in the image have changed. The minimum value estimates the most subsurface defect and the maximum estimates the sound area value. As can be seen in table 2, inspection of the planar CFRP006 specimen is not affected by non-uniform excitation and the maximum value estimates the depth reached by the heating pulse. However, for the trapezoidal CFRP008 specimen the maximum and minimum values present discrepancies which can be explain from the focus of the flashes. When two flashes are used the heating pulse is strongly focalized on the corner of the trapezoidal shape decreasing the frequency of high-value scores of distribution at the most subsurface defect. That is the distribution is most skewed to the right than when only one flash is used. The higher maximum value is related to the higher effective thickness when two flashes are used. 
Table 2 Maximum and minimum skewness parameter value for different excitations.

\begin{tabular}{|l|l|l|l|l|}
\hline Excitation & $\begin{array}{l}\text { CFRP0006 } \\
\text { maximum }\end{array}$ & $\begin{array}{l}\text { CFRP0006 } \\
\text { minimum }\end{array}$ & $\begin{array}{l}\text { CFRP0008 } \\
\text { maximum }\end{array}$ & $\begin{array}{l}\text { CFRP0008 } \\
\text { minimum }\end{array}$ \\
\hline One Flash & 7.0243 & 5.0193 & 6.9993 & 4.7193 \\
\hline Two Flash & 7.1911 & 5.0173 & 8.0441 & 5.3386 \\
\hline
\end{tabular}

\section{Impact of the number of thermograms used in the skewness image.}

Skewness is a statistic parameter of a distribution; therefore it depends on the population of the distribution. In order to determinate the dependence of the skewness image with respect to the number of thermograms included in the calculations six progressive windowing sizes of recorded thermograms were used. Figure 7 shows the skewness image obtained using 300,600,900, 1200, 1500 and 1800 thermograms. Decreasing the number of thermograms for the calculation of the skewness image reduces the population of scores in the left of the distribution, as well as the skewness value for all surface points. Therefore, the number of thermograms to be included in the estimation of the skewness parameter must be large enough as to reach thermal equilibrium after the pulsed excitation. In order to determinate when this condition is reached, the skewness image contrast is defined as the ratio between the minimum and the maximum value in the skewness image.

Based on the stability of the skewness image contrast and the number of detected defect, it can be concluded that a minimum windowing size of 1200 thermograms is required for the calculation of the skewness image (see Table 3).

Table 3 Result obtained to the skewness parameter measure at CPRP006 for different frames number in temporal windows.

\begin{tabular}{|l|c|c|c|c|}
\hline $\begin{array}{l}\text { Frames } \\
\text { number }\end{array}$ & $\begin{array}{l}\text { Maximum } \\
\text { Skewness value }\end{array}$ & $\begin{array}{l}\text { Minimum } \\
\text { Skewness value }\end{array}$ & $\begin{array}{l}\text { Detected defect } \\
\text { number }\end{array}$ & $\begin{array}{l}\text { Skewness Image } \\
\text { contrast }^{1}\end{array}$ \\
\hline 300 & 3.2870 & 2.1117 & 17 & 0,64 \\
\hline 600 & 4.3127 & 2.8971 & 15 & 0,67 \\
\hline 900 & 5.1823 & 3.5317 & 20 & 0,68 \\
\hline 1200 & 5.9363 & 4.0859 & 23 & 0,69 \\
\hline 1500 & 6.5979 & 4.5751 & 23 & 0,69 \\
\hline 1800 & 7.1911 & 5.0173 & 23 & 0,7 \\
\hline
\end{tabular}

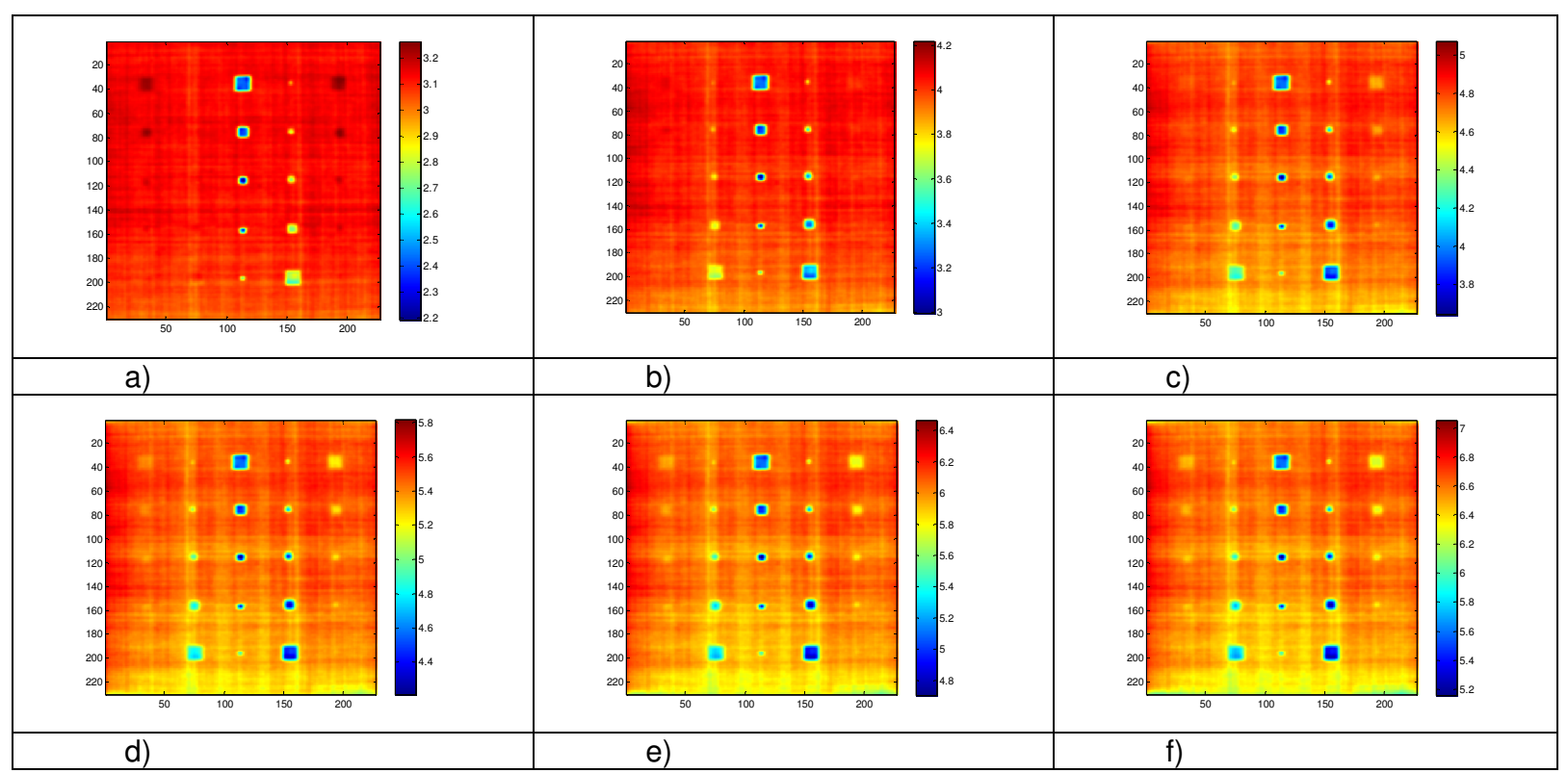

Fig 7. Unique skewness parameter image for different frames number in temporal windows size a) 300 ,

\section{Conclusions}

b) 600, c) 900 , d) 1200 , e) 1500 , f) 1800 thermograms

The proposed method based on the skewness statistic parameter promises to be a powerful tool for detecting subsurface defect by active thermography. The skewness parameter calculation can be seen as a process to compress the thermographic sequence into a unique image, which exhibits an improved contrast.

\footnotetext{
${ }^{1}$ Minimum Skewness value/maximum Skewness value.
} 
Furthermore, the process of defect detection in pulsed thermography is completely automated. Experimental works using CFRP specimens were performed demonstrating that the skewness parameter applied to thermographic sequences is practically unaffected by non-uniform excitation ( 1 and 2 flashes) and surface shape (planar, curved and trapezoidal). Summarizing, the proposed technique provides an automatic method that can be used to produce compressed data (in a unique image) from large 3D matrices typical of active thermography experiments.

\section{Acknowledgments}

This work was supported by the Spanish Science and Technology Minister under project TEC2007-67987C02-01 and Jose Castillejo program, the Chaire de recherche du Canada (MiViM) and the Ministère du développement économique, innovation et exportation du Québec.

\section{REFERENCES}

[1] X. P. V. Maldague, Theory and Practice of Infrared Technology for Nondestructive Testing, 1 edition ed.: Wiley-Interscience, April 2001.

[2] S. M. Shepard, J. R. Lhota, B. A. Rubadeux, D. Wang, and T. Ahmed, "Reconstruction and enhancement of active thermographic image sequences," Optical Engineering, vol. 42, pp. 1337-42, 2003.

[3] D. A. Gonzalez, C. Ibarra-Castanedo, F. J. Madruga, and X. Maldague, "Differentiated absolute phase contrast algorithm for the analysis of pulsed thermographic sequences," Infrared Physics \&amp; Technology, vol. 48, pp. 16-21, 2006.

[4] X. Maldague and S. Marinetti, "Pulse phase infrared thermography," Journal of Applied Physics, vol. 79, p. 2694, 1996.

[5] N. Rajic, "Principal component thermography for flaw contrast enhancement and flaw depth characterisation in composite structures," Composite Structures, vol. 58, pp. 521-8, 2002.

[6] A. Azzalini and A. D. Valle, "The multivariate skew-normal distribution," Biometrika, vol. 83, pp. 715-726, December 1, 19961996.

[7] W. L. Hays and R. L. Winkler, Statistics: Probability, inference, and decision. New York: Holt, Rinehart \& Winston., 1971.

[8] "Thermocal 3D Software manual," in Institute of Introscopy, Tomsk Polytechnic, Russia, 1998. 